\title{
COMPLEX GEOMETRY AND OPERATOR THEORY ${ }^{1}$
}

\author{
BY M. J. COWEN ${ }^{2}$ AND R. G. DOUGLAS
}

Communicated by I. M. Singer, September 1, 1976

One of the principal goals of spectral theory for operators is to find unitary invariants which are local relative to the spectrum. Multiplicity theory provides a complete set of such invariants for normal operators on (complex) Hilbert space. For general operators on finite-dimensional Hilbert space a nilpotent operator is attached to each point of the spectrum and these "local operators" together with their relative location provide a complete set of unitary invariants. In this note we announce analogous results for a class of operators whose characteristic property is having an open set of eigenvalues. Included in this class are the backward shift together with the adjoint of various subnormal, hyponormal, and weighted shift operators. Although our goal is to provide a systematic spectral theoretic approach to the study of this type of operator, here we are concerned only with a result on unitary equivalence.

For $\Omega$ a connected open subset of $C$ and $n$ a positive integer, let $B_{n}(\Omega)$ denote the (bounded linear) operators $T$ defined on the separable Hilbert space $H$ which satisfy: (1) $\Omega$ is contained in the spectrum $\sigma(T) ;(2)(T-\omega) H=H$ for $\omega$ in $\Omega$; (3) $\operatorname{dim} \operatorname{ker}(T-\omega)=n$ for $\omega$ in $\Omega$; and (4) $\bigvee_{\omega \in \Omega} \operatorname{ker}(T-\omega)=$ $H$. To study $T$ in $B_{n}(\Omega)$ we introduce the "local operators" $N_{\omega}$ on $N_{\omega}$ defined for each $\omega$ in $\Omega$, where $N_{\omega}=\operatorname{ker}(T-\omega)^{n+1}$ and $N_{\omega}=(T-\omega) \mid N_{\omega}$. Observe that $N_{\omega}$ is nilpotent of order $n+1$ on a space of dimension $n(n+1)$. Our principal result is

THEOREM 1. Operators $T$ and $T^{\prime}$ in $B_{n}(\Omega)$ are unitarily equivalent if and only if $N_{\omega}$ is unitarily equivalent to $N_{\omega}^{\prime}$ for $\omega$ in $\Omega$.

REMARKS. (1) For $n=1$ the real analytic function $\operatorname{tr} N_{\omega} N_{\omega}^{*}$ is a complete set of unitary invariants. In general, the complete set of unitary invariants can be chosen to be real analytic functions defined as the traces of a finite number of words in $N_{\omega}$ and $N_{\omega}^{*}$.

(2) For "generic operators" one need only consider $(T-\omega) \mid \operatorname{ker}(t-\omega)^{3}$.

(3) Comparatively trivial results obtain if one allows knowledge of either $(T-\omega) \mid \operatorname{ker}(T-\omega)^{k}$ or $T \mid \bigvee_{i=1}^{k} \operatorname{ker}\left(T-\omega_{i}\right)$ with $\omega_{1}, \omega_{2}, \ldots, \omega_{k}$ in $\Omega$ for arbitrary $k$.

AMS (MOS) subject classifications (1970). Primary 47A10, 47 B20, 53B35.

${ }^{1}$ Supported by grants from the National Science Foundation.

${ }^{2}$ Sloan Foundation Fellow and SUNY-Research Foundation Fellow. 
We are able to pass from local data to a global description by using complex geometry. The map $\omega \rightarrow \operatorname{ker}(T-\omega)$ can be shown to define a holomorphic mapping $\varphi_{T}$ from $\Omega$ to the Grassmannian $\operatorname{Gr}(n, H)$ and hence the pullback defines a hermitian holomorphic bundle $E_{T}$ with real analytic metric over $\Omega$. Two such bundles $E$ and $E^{\prime}$ over $\Omega$ are said to be equivalent if there is a biholomorphic bundle map which is an isometry from each fiber $E_{\omega}$ onto $E_{\omega}^{\prime}$. That $E_{T}$ determines $T$ up to unitary equivalence follows from a generalization of Calabi's rigidity theorem [1]:

THEOREM 2. Holomorphic maps $\varphi$ and $\varphi^{\prime}$ from $\Omega$ into $\mathrm{Gr}(n, H)$ define equivalent pullback bundles if and only if $\varphi=U \varphi^{\prime}$ for some unitary operator $U$ on $H$.

Now we must consider the equivalence problem for the bundles which arise from Grassmannians (cf. [4] for the case $G r\left(n, \mathbf{C}^{2 n}\right)$ ). With no additional difficulty we consider arbitrary hermitian holomorphic bundles over $\Omega$ with real analytic metric. Since such a bundle has a canonical connection defined on it, and hence a curvature form, we are considering a special case of the classical equivalence problem in geometry studied earlier by Veblen, E. Cartan and others (cf. [5]). Our contribution is

THEOREM 3. If $E$ and $E^{\prime}$ are hermitian holomorphic bundles of rank $n$ with real analytic metric defined over a simply connected $\Omega$, then there exists an integer $0 \leqslant k \leqslant n$ such that $E$ and $E^{\prime}$ are equivalent if the $(p, q)$-covariant derivatives of the curvatures, $K_{z} p_{\bar{z}} q$ and $K_{z}^{\prime} p_{\bar{z}} q$, of $E$ and $E^{\prime}$, respectively, for $\{(p, q): p+q \leqslant k ; p, q \neq k\}$ are simultaneously unitarily equivalent at each $\omega$ in $\Omega$.

Although general results on this type of equivalence problem do state the existence of an integer $k$ for which the preceding result is valid, we believe none provides this upper bound on $k$ for the case of hermitian holomorphic bundles. Moreover, our techniques yield the same result in the case of $C^{\infty}$ metric off a nowhere dense closed set.

If the two bundles $E$ and $E^{\prime}$ are obtained as pullbacks of holomorphic maps from $\Omega$ into Grassmannians, then the theorem holds without assuming $\Omega$ is simply connected.

For generic bundles second order derivatives suffice; here generic means that the eigenvalues of the curvature at some point are distinct and of multiplicity one. In a preliminary exposition [2] we believed that second order invariants would always suffice. Although we have been unable to construct examples to the contrary, we now doubt this.

Complete details including many more ancillary results both in complex geometry and operator theory will appear in [3]. 


\section{BIBLIOGRAPHY}

1. E. Calabi, Isometric imbedding of complex manifolds, Ann. of Math. (2) 58 (1953), 1-23. MR 15, 160.

2. M. J. Cowen and R. G. Douglas, Operator theory and complex geometry, Proc. Sympos. Pure Math., vol. 30, part 2, Amer. Math. Soc., Providence, R.I. (to appear).

3. - Operator theory and complex geometry, Advances in Math. (to appear).

4. P. A. Griffiths, On Cartan's method of Lie groups and moving frames as applied to uniqueness and existence questions in differential geometry, Duke Math. J. 41 (1974), $775-814$.

5. M. Kuranishi, On E. Cartan's prolongation theorem of exterior differential systems, Amer. J. Math. 79 (1957), 1-47. MR 18, 474.

DEPARTMENT OF MATHEMATICS, STATE UNIVERSITY OF NEW YORK AT STONY BROOK, STONY BROOK, NEW YORK 11794 